\title{
Relevance of Instruments for Measurements of Quality of Life in the AMNOG Context: An Analysis of the Endpoint Health-Related Quality of Life from Different Perspectives

Nadine Schneider

\begin{abstract}
In the assessment of the additional therapeutic benefit of newly approved drugs, different patient-relevant outcomes are used, such as Health-related Quality of Life (HrQoL). Among the four outcomes (morbidity, mortality, adverse effects), $\mathrm{HrQoL}$ is the most subjective and also a relatively new outcome. This thesis focuses on the relevance of HrQoL data within the AMNOG process, including available instruments for the measurement of HrQoL. Moreover, interviews were conducted to investigate different perspectives regarding the relevance of this endpoint in comparison to the others. Until now, HrQoL is perceived more as a supportive tool rather than an equivalent criterion. Possible reasons for that are technical limitations (response rate, validated questionnaires) as well as differences with regard to the profile of requirements for the pharmaceutical company concerning the market authorization (study design).
\end{abstract}

\section{Relevance of Instruments for Measurements of Quality of Life in the AMNOG Context}

\subsection{An Analysis of the Endpoint Health-Related Quality of Life from Different Perspectives}

Since the Act on the Reform of the Market for Medicinal Products (AMNOG) came into effect, the pharmaceutical companies have been facing an additional burden with regard to the reimbursement negotiations with the statutory health insurances.

\footnotetext{
N. Schneider $(\bowtie)$

Fraunhofer Institute for Molecular Biology and Applied Ecology IME, Branch for Translational Medicine and Pharmacology TMP, Frankfurt am Main, Germany

e-mail: Nadine.Schneider@ime.fraunhofer.de
} 
Manufacturers are free to set their price for a newly approved drug in the first year after market access. Since 2011, they need to prove the additional therapeutic benefit of the new drug in order to negotiate the reimbursement price according to the therapeutic value (Wenzl and Paris 2018). The political goal was saving the sick funds 2.2 billion $€$ per year by stemming the rapidly increasing pharmaceutical expenditures (Leverkus and Chuang-Stein 2016). Moreover, it was introduced to create a fair competition and to focus more on the patients' well-being.

The assessment of an additional therapeutic benefit is based on patient-relevant outcomes, such as mortality, morbidity and Health-related Quality of Life (HrQoL) in comparison to the standard of care. The level of additional benefit in comparison to an appropriate comparative therapy is categorized as: major, significant, marginal, not quantifiable, none or less (Leverkus and Chuang-Stein 2016). The choice of comparator is very crucial for the pharmaceutical company and it is determined by the G-BA (Federal Joint Committee), which is the highest decision making body. The decision is based on label and medical guidelines, the criteria for determining the appropriate comparator is written in G-BA's rules of procedure (G-BA 2018).

The added benefit, determined by the G-BA, is highly beneficial for the pharmaceutical company with regard to the reimbursement negotiations with the SHI. If the G-BA finds that the drug does not have any additional benefit, the SHI will pay no more than what the equivalent products (often generic drugs) already cost. Thus, the company's goal is to show an added benefit and a higher value of their newly launched drug compared to the appropriate comparative therapy to achieve a sustainable reimbursement price. In general, this requires a strategic rethinking from the pharmaceutical companies with regard to their market access strategy. They will have to learn to live with an unpredictable market access process (Sieler et al. 2015).

Among the four outcomes, $\mathrm{HrQoL}$ is the most subjective and also a relatively new outcome (Blome et al. 2017). The use of patient reported outcomes (such as HrQoL) in comparative effectiveness research can be challenging, for instance when it comes to the selection of appropriate instruments or interpretation of results. HrQoL data are difficult to obtain, complex and subjective. Furthermore, there are technical limitations with regard to the different questionnaires available. The G-BA only accepts certain validated questionnaires for the assessment of an additional benefit.

This master thesis focuses on the analysis of the outcome category Health related Quality of Life (HrQoL) by evaluating the different perspectives of the involved parties. The goal is to analyze the importance of $\mathrm{HrQoL}$ data and their significance for patient-relevance within the AMNOG process. What does an improvement of HrQoL means for a patient in a certain indication? Moreover, how do IQWiG and G-BA evaluate HrQoL? Is this endpoint a fully equivalent outcome category within the assessment process? 


\section{Methods}

To analyze the relevance of HrQoL data in the assessment of an additional benefit, two major strategies were pursued:

1. The primary focus of this master thesis lays on the assessment of drugs in rheumatic indications. Therefore, five newly (2015 until 2017) approved drugs in the indication areas psoriasis, psoriatic arthritis and rheumatoid arthritis were chosen and analyzed to find out whether HrQol data were included in the dossier or not. Moreover, it was documented if G-BA and IQWiG accepted the data and if these data were crucial for the granting of an additional benefit.

2. To investigate potential differences regarding the relevance of HrQoL data within the AMNOG process, interviews with different parties were conducted: With a representative from a pharmaceutical company, vfa (Verband Forschender Arzneimittelhersteller, Association of Research-Based Pharmaceutical Companies) and with a rheumatologist/clinician. Furthermore, definitions and statements published by G-BA and IQWiG will be provided to represent the view of the regulatory authorities. This master thesis is a qualitative approach rather than a quantitative one. The goal was to get a comprehensive picture about the relevance of HrQoL data and the used instruments with their strengths and weaknesses within the AMNOG process.

\section{Results and Conclusions}

It seems that the endpoint HrQoL is still the most difficult endpoint to have in the benefit assessment. This is because of several reasons: Firstly, HrQoL data are based on a subjective assessment. Secondly, especially validated instruments used for the measurement are limited. Thirdly, it seems that HrQoL data are experienced rather as a supportive tool than as an equivalent criterion.

For the assessment of HrQoL patients are asked to fill in different questionnaires about their well-being or pains. Each person experiences pain in a different kind of way and in a different intensity. Moreover, the experience of pain can be influenced by different factors e.g. mental state or the personal attention of the doctor. Thus, it is very difficult to get an objective assessment about the change of HrQoL caused by a certain medication. How well represented is the change of HrQoL through this kind of questionnaires? The answer to this question is of course dependent on the indication and also on the available disease-specific questionnaires. Nevertheless, the goal of validated questionnaires is to transform a subjective opinion into an objective evaluation that quantifies the patient perception. The three main properties for the determination of outcome questionnaires are objectivity, reliability (reproducibility) and validity (content, construct, criterion) (Hamilton et al. 2017). 
Difficulties also exist with regard to technical and/or organizational limitations such as low response rate or the lack of validated and suitable questionnaires. G-BA and IQWiG do not accept HrQoL data with a response rate lower than $80 \%$. This can be challenging to achieve, depending on the indication. In rheumatic diseases, patients may not be able to fill in the form properly because their hand and finger joints hurt. Generally speaking, pain is often the reason why patients are not able to fill in the questionnaire, this being also true for other indications such as cancer. Another reason can be that the patients suffer from depression and thus are not willing to fill in the form. Sometimes the self-assessment is impaired by the disease, e.g. in case of schizophrenia and Alzheimer's disease. Furthermore, elderly people often have difficulties with that task. Some also report that some of the questionnaires are too long or that they need to fill in several ones. There are many reasons for a low response rate which are challenging to overcome. With respect to G-BA and IQWiG a low response rate leads to invalid data and a possible imbalance between the study arms, thus the data will be excluded from the assessment.

Apart from the response rate, another problem is the lack of validated and suitable questionnaires. IQWiG requests a generic and a disease-specific questionnaire for the assessment of HrQoL. However, depending on the indication, there is often no validated disease-specific questionnaire so far. In the dossiers submitted for RA drugs, the pharmaceutical company only uses the SF-36, which is a generic tool. It seems that this questionnaire works quite well for the assessment of the general health status, but there is no single question included about disease-specific problems. Patients with RA often suffer from pain in the joints. Since mostly the fingers are impaired, they are not able to get dressed or cook. For instance, they need a special solution to open a bottle of water, which often means that they need help in their daily routine. This is different for PsO and PsA, since in these two indications the pharmaceutical company mostly uses the DLQI (Dermatology Life Quality Index) questionnaire. This is a disease-specific form that addresses 10 questions with regard to skin specific problems e.g. "... how much has your skin affected any social or leisure activities?" An assessment of an improvement of HrQoL works quite well for these two indications. The introduction of new disease-specific instruments means a big effort and is not that easy to implement. A new questionnaire needs to be validated with regard to reliability, internal consistency, unidimensionality and responsiveness. Moreover, the language availability needs to be considered. Most clinical studies are international and thus the questionnaire needs to be available in a large number of languages, which means a big effort in terms of time and money (Doward et al. 2004).

It seems that $\mathrm{HrQoL}$ data are still perceived rather as a supportive tool than as an equivalent outcome. Especially in rheumatic indications, other outcomes have much more relevance than $\mathrm{HrQoL}$ within the assessment process. That can also be due to the difficulties to evaluate the clinical relevance of potential differences. What is meaningful for the patient? The minimal clinically important difference (MCID) is defined as the minimal differences in scores of an outcome measure that is perceived by patients as beneficial or harmful (Keurentjes et al. 2012). 
Apparently the relevance of HrQoL data is higher in oncological disease where the disease caused a significant reduction of lifetime. In these kind of indications a substantial improvement of HrQoL, whereas morbidity and mortality are the same as with the appropriate comparative therapy, will be enough for a major added benefit (Hecken 2016).

One of the major problems is the different profile of requirements for the pharmaceutical company. For the market authorization, the focus lies on a reasonable benefit/risk ratio, which means that while designing the clinical trials the outcomes mortality, morbidity and adverse effects are much more important. HrQoL is so far no criterion within the market authorization process. Very often the trials are big international studies, which means a big effort recruiting the patients. Primarily the pharmaceutical company's goal is to obtain market authorization in the preferred countries, which also means dealing with different authorities at the same time. The pharmaceutical company is focused on fulfilling the requirements of EMA and/or FDA and that means a lot of strategic planning before running the clinical trial. The second priority is the benefit assessment in Germany where the focus is more on a patient-relevant added benefit including the aspect HrQoL data. This situation forces the pharmaceutical company to plan the clinical trials in accordance with EMA/FDA for the market authorization, but at the same time the pharmaceutical company also needs to plan the clinical trials for G-BA/IQWiG and a lot of other HTA bodies in Europe and outside from Europe to obtain a beneficial assessment for the reimbursement negotiations with the health insurances. Since the requirements differ in certain aspects, this is a really challenging and costly situation. That could prospectively force the pharmaceutical companies to rethink their market access strategy. It will get more unattractive aiming for a market authorization in Germany, if the AMNOG process is not going to improve. However, since Germany is one of the biggest markets (fourth largest worldwide) (Bütow 2017) in the pharmaceutical sector, this will not happen that fast.

Moreover, the AMNOG process could also, to a certain extent, influence the decision about which indication area it is worth to invest in concerning research activity. In some cases even really innovative new treatment options do not obtain the appropriate added benefit, which would enable the company to amortize their investment/expenses. It is not worthwhile for a company to invest in a drug which will be compared to a generic standard of care.

\section{Outlook}

Today HrQoL is not an equivalent criterion in the assessment of an added benefit of new drugs. However, the importance and the relevance to include such data have been increasing over time. The authorities have already started to sanction the lack of HrQoL data, at least for certain indications (Hecken 2016). The relevance to include sufficient HrQoL data is getting more important for the pharmaceutical company as well. To overcome the limitations the pharmaceutical company is facing, especially 
with regard to different requirements each authority demands, the set of rules needs to be adapted.

IQWiG and G-BA should be more flexible and willing to adapt to individual solutions. An example: On the one hand, the ministry of health wants to support research in the field of new antibiotics. But on the other hand, this research is not worth financially for the pharmaceutical companies since the added benefit will most probably be compared to a generic product. The pharmaceutical companies have no chance to amortize their expenses. To solve this problem, an individual solution needs to be implemented like in the case of orphan drugs.

It would be desirable that the whole AMNOG process gets more pragmatic with regard to the medical daily practice. Partly the required high numbers of patients, which should be included in the clinical trials, are not realistic and are difficult to fulfill. Furthermore, the requested duration of the clinical trials sometimes makes no sense with regard to the mode of action of the new drug. An artificial prolongation of the study duration only to fulfill the requirements is not only very costly for the pharmaceutical company but also doubtful in terms of ethics, especially for the patients in the placebo arm. Individual and disease-specific solutions are needed here.

Speaking about HrQoL data, the question is which time frame is the optimal one for the assessment of an increase of HrQoL? In indications where depression or other perceptual disorders are increased, this could also influence the self-assessment and the assessment of their well-being. This would then lead to a non representative picture of the HrQoL status. It should be possible to adapt the design of the clinical trial in accordance with the specific requirements for the assessment of HrQoL data.

An individual adaptation of the study design only makes sense if validated and well-designed disease-specific questionnaires exist. And this for every indication. Moreover, it would be advantageous if questionnaires, which are accepted for EMA and FDA, are also accepted by IQWiG and G-BA, such as the EQ-5D form. Generally speaking, more flexibility concerning the choice of questionnaires and a greater alignment to the requirements needing to be fulfilled to obtain market authorization would be preferable.

Taken all these considerations together, the AMNOG process needs to be further developed, both for the assessment of HrQoL data and with regard to the general very technical-driven requirements. It is indeed important to regulate the price system in the pharmaceutical market, but with a certain dose of common sense and an understanding of what is important for the patients and for the well-being of the whole population. The pharmaceutical market still has a big medical need of new innovative and "cost-intelligent" drugs. The AMNOG process should not interfere with the devolvement of innovation in Germany.

There are also further developments with regard to the market access sector, which will influence the conduction of clinical trials. There will be a new Clinical Trial Regulation in 2019, which means a major change concerning the conduction of clinical trials. The new Regulation (Clinical Trial Regulation EU No. 536/2014) has the goal to harmonize the assessment and supervision process for clinical trials throughout the EU (European Medicine Agency 2014). This Regulation will repeal 
the existing EU Clinical Trial Directive (EC) No. 2001/20/EC. The key benefits of the regulation are harmonized electronic submission and assessment process for clinical trials conducted in multiple member states, improved collaboration with regard to information-sharing and decision-making. Furthermore, an increased transparency of information on clinical trials and the highest standard of safety for all participants are also of importance (European Medicine Agency 2018). Similar efforts to improve the conduction of clinical trials are seen by the FDA (Food and Drug Administration US 2018). Changes regarding the planning and conduction of clinical trials will automatically influence future Health Technology Assessments. The impact of these changes on the outcome category HrQoL and its consequent implementation in the trial design will be seen and further analyzed in the future.

\section{References}

Blome, C., Augustin, M., Metin, H., \& Lohrberg, D. (2017). Four years of early benefit assessment of new drugs in Germany: A qualitative study on methodological requirements for quality of life data. The European Journal of Health Economics, 18(2), 181-193. https://doi.org/10.1007/ s10198-016-0765-6.

Bütow, S. M. (2017, March). Industry overview: The pharmaceutical industry in Germany. Retrieved from https://www.vfa.de/embed/the-pharmaceutical-industry-in-germany.pdf

Doward, L. C., Meads, D. M., \& Thorsen, H. (2004). Requirements for quality of life instruments in clinical research. Value in Health, 7, S13-S16. https://doi.org/10.1111/j.1524-4733.2004. 7s104.x.

European Medicine Agency. (2014). Clinical Trial Regulation EU No. 536/2014. Official Journal of the European Union (Vol. 2014). https://doi.org/http://eur-lex.europa.eu/pri/en/oj/dat/2003/1_ 285/1_28520031101en00330037.pdf

European Medicine Agency. (2018). Clinical Trial Regulation. Retrieved September 23, 2018, from http://www.ema.europa.eu/ema/index.jsp?curl=pages/regulation/general/general_con tent_000629.jsp\&mid=WC0b01ac05808768df

Food and Drug Administration US, R. P. (2018). FDA advances efficient approaches to designing and conducting cancer clinical trials. Retrieved September 23, 2018, from https://blogs.fda. gov/fdavoice/index.php/2018/08/fda-advances-efficient-approaches-to-designing-andconducting-cancer-clinical-trials/

G-BA. (2018). Verfahrensordnung. Retrieved from https://www.g-ba.de/downloads/62-492-1614/ VerfO_2018-03-16_iK-2018-07-05.pdf

Hamilton, D. F., Giesinger, J. M., \& Giesinger, K. (2017). It is merely subjective opinion that patient-reported outcome measures are not objective tools. Bone \& Joint Research, 6(12), 665-666. https://doi.org/10.1302/2046-3758.612.BJR-2017-0347.

Hecken, J. P. (2016). Lebensqualität und Patientennutzen - Konsequenzen für die Nutzenbewertung. Frankfurter Forum - Diskurse. Retrieved from http://frankfurterforumdiskurse.de/wp-content/uploads/2016/11/Heft_14_Vortrag_4.pdf

Keurentjes, J. C., Van Tol, F. R., Fiocco, M., Schoones, J. W., \& Nelissen, R. G. (2012). Minimal clinically important differences in health-related quality of life after total hip or knee replacement: A systematic review. Bone \& Joint Research, 1(5), 71-77. https://doi.org/10.1302/20463758.15.2000065.

Leverkus, F., \& Chuang-Stein, C. (2016). Implementation of AMNOG: An industry perspective. Biometrical Journal [Biometrische Zeitschrift], 58(1), 76-88. https://doi.org/10.1002/bimj. 201300256 
Sieler, S., Rudolph, T., Brinkmann-Saas, C., \& Sear, R. (2015). AMNOG revisited. Retrieved from https://www.mckinsey.com/industries/pharmaceuticals-and-medical-products/our-insights/ amnog-revisited

Wenzl, M., \& Paris, V. (2018, June). Phamaceutical reimbursement and pricing in Germany, 1-22. Retrieved from http://www.oecd.org/els/health-systems/Pharmaceutical-Reimbursement-andPricing-in-Germany.pdf

Open Access This chapter is licensed under the terms of the Creative Commons Attribution 4.0 International License (http://creativecommons.org/licenses/by/4.0/), which permits use, sharing, adaptation, distribution and reproduction in any medium or format, as long as you give appropriate credit to the original author(s) and the source, provide a link to the Creative Commons licence and indicate if changes were made.

The images or other third party material in this chapter are included in the chapter's Creative Commons licence, unless indicated otherwise in a credit line to the material. If material is not included in the chapter's Creative Commons licence and your intended use is not permitted by statutory regulation or exceeds the permitted use, you will need to obtain permission directly from the copyright holder.

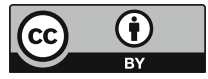

